\title{
Conflictos sociales urbanos en Lima: la centralidad de la comunicación en las disputas en torno a la residencialidad*
}

\author{
Sandro Macassi \\ Pontificia Universidad Católica del Perú \\ semacassi@pucp.pe
}

Recibido: 1/8/2018 / Aceptado: 23/10/2019

doi:10.26439/contratexto2019.n031.3893

\begin{abstract}
Resumen. Este estudio se basó en el análisis de tres short cases sobre conflictos en que los vecinos eran afectados en su residencialidad debido a proyectos de gobiernos locales o empresas que buscaban intensificar y diversificar el uso del espacio urbano. Se entrevistó a las partes primarias involucradas en la disputa y se revisó información secundaria; además se hizo una revisión de fuentes secundarias periodísticas, medios sociales y publicaciones oficiales para construir los casos. Los grupos de residentes emplearon medios digitales para generar cohesión interna, implementar sistemas de alerta temprana y difundir sus posiciones. Los gobiernos locales evidenciaron una carencia de estrategias comunicativas para distender el conflicto o construir confianza; en cambio se priorizó una estrategia de confrontación, poco transparente, que acrecentó la polarización y la oposición. Los repertorios comunicativos empleados por residentes y autoridades se concentraron en fortalecer su posición y poder en el conflicto, buscando impactar en otros actores para tener un mayor respaldo público a sus causas. Por otro lado, fueron escasos los recursos comunicativos empleados para construir puentes, generar acercamientos y procesos de diálogo. Hace falta reenfocar la dimensión comunicativa en la gestión de los conflictos urbanos, especialmente desde la acción gubernamental.
\end{abstract}

Palabras clave: comunicación y conflictos / transformación de conflictos / conflictos urbanos

Este artículo fue posible gracias a los estudios parciales desarrollados para los tres casos por los y las alumnas del curso de Comunicación y Conflictos de la Pontificia Universidad Católica del Perú (PUCP), del primer y segundo semestre del 2016: María José Arguedas, Carmen Alarco, Hana Quirós, Alessandra Contreras y Alonso Quiroz; Eva Benites, Daniela Cuba, Nedelka Tamariz, Pamela Vivar, Erwin García, Claudia Santiago, Ivanoei Carrasco y Mayra Vidal. 


\title{
Urban social conflicts in Lima: the centrality of communication in conflicts around the residential neighborhood character
}

\begin{abstract}
Авstract. This study was based on the analysis of three short cases of conflicts where the residential neighborhood character of an area was affected due to projects conducted by local governments or companies which sought to intensify and diversify the use of the urban space. The primary parties involved in the conflict were interviewed and secondary data was analyzed. Additionally, secondary journalistic sources, social media and official publications were reviewed to build the cases. The groups of residents used digital media to generate internal cohesion, implement early warning systems and spread their points of view. Local governments showed a lack of communicative strategies to ease the conflict or build trust. Instead, priority was given to a confrontational and non-transparent strategy that increased polarization and opposition. The communicative repertoires used by residents and authorities focused on strengthening their points of view and power in the conflict, aiming to have an impact on other actors to get greater public support for their cause. Furthermore, the communication resources used to build bridges, and generate rapprochement and dialogue were poor. Refocusing on the communication dimension for managing urban conflicts is needed especially from the government side.
\end{abstract}

Keywords: communication and conflicts / conflict transformation / urban conflicts 


\section{Introducción}

$\mathrm{E}$ n Latinoamérica y en el Perú surgieron en las últimas décadas cientos de conflictos socioambientales debido al incremento exponencial de las inversiones en agroexportación, infraestructura y megaproyectos extractivos. La inversión intensiva de empresas extractivas transnacionales en los territorios de comunidades campesinas e indígenas generó alteraciones del orden social, económico y político de estas sociedades. Asimismo, los conflictos sociales derivados impactaron la gobernabilidad nacional y territorial pues, en el Perú, entre el 2006 y el 2015 se produjeron 131 fallecidos y 2312 heridos (Defensoría del Pueblo, 2017) y generaron la caída de tres gabinetes presidenciales. Sin embargo, las inversiones también se multiplicaron en las urbes debido a que países de renta baja como el Perú, pasaron a ser países de renta media, ampliando la clase media que, de la mano del crecimiento demográfico, impulsaron en la ciudad de Lima una mayor presión por viviendas bajo un esquema de crecimiento vertical.

Lima tuvo por muchas décadas un lento proceso de expansión horizontal, pero conforme los terrenos se fueron acabando, el boom constructor y las políticas neoliberales urbanas plantearon un esquema de crecimiento vertical en los distritos más acomodados (Yáñez, 2014). A raíz de esto, mucha de la infraestructura urbana quedó obsoleta o la demanda de servicios creció exponencialmente al mismo tiempo que el incremento en los ingresos de las clases medias demandó mejores sistemas de transportes estacionamientos, servicios de agua y desagüe, pero también servicios de recreación y centros de convenciones, etcétera.

En el Perú, entre el 2006 y el 2016, el boom constructor, dinamizó la economía y fue el motor interno que, de la mano de la exportación de commodities, impulsó el crecimiento económico nacional. Sin embargo, la mayoría de los estudios sobre la conflictividad se centró en los conflictos socioambientales emblemáticos, pero muy poco se analizó cómo los cambios y transformaciones en las ciudades vienen generando procesos conflictivos de "modernización acelerada" que alteran las relaciones y prácticas tradicionales de los vecinos en poco tiempo, generando una condición de desequilibrio que según Mitchel (2010) es una de las fuentes de los conflictos (aunque no los determina). Por ello, esta investigación se enfocará en los conflictos de baja intensidad entre los vecinos que vieron afectados sus hábitats, alteradas sus costumbres y su tranquilidad, debido a que muchos de estos cambios implicaban una transformación del uso social del espacio público.

Nuestro punto de partida es el análisis y transformación de conflictos que define a los conflictos sociales como la percepción supuesta o real de una o ambas partes de que el acceso a los recursos y la satisfacción de las necesidades básicas (Rubenstein, Botes, Dukes y Stephens, 1994; Burton, 2000) se ven amenazados por los objetivos de la otra parte, conformándose una incompatibilidad mutua de 
intereses (Kriesberg y Dayton, 2012; Mitchell, 2016), lo cual conduce al desarrollo de acciones para la predominancia de sus objetivos sobre los de la otra parte (Pruitt, Rubin y Kim, 2004) desde un marco cultural (Avruch, 2013).

Esta perspectiva pone atención en los procesos de relacionamiento entre las partes (Huamaní, Macassi, Alegría y Rojas, 2012), analizando las acciones tanto de diálogo como coercitivas, desplegadas por los actores como procesos de interacción que son acompañados por dinámicas sociocognitivas que le dan forma al conflicto (Vallacher et al., 2013; Deutsch y Coleman, 2012) y que deben ser analizados desde las perspectivas de ambas partes y no solo desde la narrativa de una de ellas.

Los estudios de comunicación que abordan los conflictos en Latinoamérica se centraron en la cobertura mediática (Ariñez, 2007; Díaz, Rojas y Romero, 2005; Eto, 2010; Huamán, 2012: Macassi, 2011; Torres 2007; Torrico, 2009, Sandoval, 2015: Villegas, 2007; ) pero pocos estudios analizaron los procesos comunicativos intergrupales desde una perspectiva de análisis y transformación de conflictos. Asimismo, los estudios sobre las relaciones intergrupales en los conflictos se enfocaron en el rol de la comunicación en la negociación o la intervención de terceras partes (Fisher, 2009), o en su uso en periodos de postconflicto para la prevención (Hieber, 2001; DFID, 2000), prestando poca atención a las estrategias comunicativas intergrupales de las partes para crear puentes, espacios en común y tender lazos.

Asimismo, existe otra vertiente de estudios en Latinoamérica que analiza el espacio urbano como un escenario de conflicto desde la perspectiva de los estudios culturales, donde los actores barriales desde sus discursos de apropiación del territorio confrontan las significaciones hegemónicas sobre la ciudad (García-Vargas, Gaona y López, 2016) o trabajos donde los desastres naturales son acontecimientos que generan representaciones mediáticas de la ciudad en disputa con las visiones de los actores urbanos (Reguillo, 2005) que además se encuentran en disputa con las políticas públicas sobre lo urbano a partir de los imaginarios de ciudad surgidos de sus prácticas cotidianas y su apropiación del esapcio (Silva, 2007), desarrollando en estos procesos acciones comunicacionales de autodefinición, expresión de sus visiones de mundo e intervenciones de apropiación de lo urbano (Cáneva, 2015). En muchos de estos estudios se analizan los discursos y prácticas comunicativas en contraste con los discursos hegemónicos de los medios, describiendo la conflictividad de las representaciones en disputa. Sin embargo, no se presta atención a las interacciones colaborativas y a los procesos de diálogo.

Por otra parte, en Latinoamérica primaron los estudios sobre movimientos y acciones sociales que abordan con exhaustividad las estrategias coercitivas en los procesos de "conflictividad política" (Martínez Allier, 2009; De Echave et al. 2009; Bebbington y Humphreys, 2009) basados en la teoría de movilización de recursos 
(Tilly, 2006; Della Porta y Diani, 2010) pero, generalmente narrados únicamente desde la perspectiva de los movimientos sociales. Hace falta complementar estos estudios con la perspectiva de las empresas o los gobiernos y ampliar el análisis incluyendo los procesos de visibilidad mediática y el uso de recursos digitales como elementos clave que afectan las dinámicas entre ambas partes.

La emergencia de los medios sociales ${ }^{1}$ y la predominancia del espacio mediático por sobre el político (Esser y Strömbäck, 2014) rediseñaron el tablero de juego sobre el que operan los actores sociales en conflicto, especialmente con el uso de los medios digitales (Tilly y Tarrow, 2015; Tarrow, 2012) que no solo tienen un efecto de difusión de sus ideas en los conflictos, sino que sirven para fortalecerse como grupos y ganar cohesión, pero también como lo señalan Snow y Benford (1992), para ganar legitimidad y ampliar su respaldo más allá de sus militantes.

Además, en Latinoamérica existe una amplia literatura del uso de la comunicación en procesos de incidencia, en procesos de desarrollo (Beltrán, 2006) y para el empoderamiento ciudadano y el cambio social (Alfaro, 2015; Gumucio, 2001) y en campañas de salud (Tufte, 2015), sin embargo, su foco de preocupación no es el conflicto como interacción sino la problemática narrada desde la perspectiva de una de las partes.

Las disputas en las ciudades en torno a las dimensiones simbólicas del territorio y en particular a las cosmovisiones ${ }^{2}$ sobre la residencialidad sobre la que operan los gobiernos empresas y vecinos, crecientemente vienen generando conflictos alrededor del mundo. En algunas ciudades los conflictos surgen debido a las afectaciones que el cambio del uso residencial genera en zonas depreciadas como los centros históricos (Castells, 1974, De los Ríos, 2017, Monterrubio, 2011), por el impacto de la migración (Castaño, 2009) o más recientemente el turismo a gran escala que genera en los residentes, por ejemplo en Canarias y en Barcelona (Simancas y García, 2012).

En el Perú, el surgimiento tardío del boom constructor ha generado un conjunto de impactos colaterales pues las demandas de bienes y servicios urbanos se han multiplicado debido al incremento de la densidad poblacional y al uso más intensivo del territorio que empuja a los gobiernos locales o a empresas a redefinir el

1 El concepto de medios sociales hace alusión a las redes sociales digitales que se diferencian del concepto de redes sociales interpersonales que tiene una larga tradición en las ciencias sociales. Para más detalle ver Obar y Wildman (2015).

2 Basándonos en Avruch (2013), en este texto nos referiremos a las "cosmovisiones" como el conjunto de representaciones, valoraciones y percepciones que los grupos sociales desarrollan en torno a objetos, espacios, prácticas o relaciones que son materia de disputa en los conflictos y que se vinculan directamente con los recursos empleados para satisfacer sus necesidades materiales, simbólicas o posicionales. 
uso del espacio público; en algunos casos en defensa de espacios de convivencia y encuentro, en otros para generar servicios urbanos más dinámicos y eficientes.

El caso es que este efecto colateral del boom constructor viene generando controversias entre las diferentes cosmovisiones sobre la residencialidad y la dinámica urbana que están en la base de la conflictividad urbana actual y es sobre lo que vamos a ocuparnos desde su dimensión comunicativa, analizando la relación entre gobernantes, empresas y vecinos.

\section{Metodología}

Este estudio se basó en el análisis de tres short cases de conflictividad urbana cuyo problema central era la alteración que proyectos constructivos generaban en la concepción de la residencialidad de los vecinos. En cada caso se entrevistó a las partes primarias involucradas en la disputa y a partes secundantes cuando fue necesario; además se hizo una revisión de fuentes secundarias periodísticas y de medios sociales para construir los casos (ver anexo) ${ }^{3}$. Los casos cortos revisados fueron los siguientes:

Caso 1: El cambio de zonificación en el distrito de Miraflores. La construcción de un hotel y un stripmall en un terreno dado en concesión por una entidad religiosa implicó que el municipio de Miraflores cambiara la categorización de la zona, que pasó de ser residencial a comercial, con la consecuente oposición de los vecinos residentes, lográndose que el municipio declare improcedente la petición de cambio de zonificación y generando un rediseño en el proyecto.

Caso 2: El proyecto del municipio de Magdalena de ampliación de los carriles en el Jirón Félix Dibós generó una resistencia de parte de los vecinos de la calle, preocupados por la pérdida de su residencialidad y la reducción de las áreas verdes. Finalmente, el proyecto amplió uno de los carriles, pero no pudo culminar el último carril debido a la fuerte oposición de los vecinos.

Caso 3: A iniciativa del Gobierno local, se desarrolló un proyecto de remodelación de la calle Los Libertadores (distrito de San Isidro) que comprendía la incorporación de áreas verdes, sembrado de árboles, restricción del tráfico, desarrollo de una ciclovía, proyecto que generó una resistencia de los vecinos, quienes veían amenazado su acceso a los estacionamientos y su residencialidad. El municipio cerró la calle para la remodelación por ocho meses e inició una campaña comunicativa de convencimiento sobre los beneficios del proyecto.

3 El anexo puede consultarse en el siguiente enlace: https://rive.google.com/file/d/1jaerNz0Xnacu4uLtmOcH-LBbbDeTCD6/view 
El análisis se basó en el enfoque de transformación de conflictos planteado por Lederach (1992), Mitchell (2016) y Kriesberg y Dayton (2012), entre otros. Se analizaron, además, las prácticas y procesos comunicativos involucrados en la conflictividad, así como los recursos comunicativos empleados en las dinámicas del relacionamiento entre las partes. Asimismo, la investigación se guió por algunas de las premisas fundamentales sobre el análisis de los conflictos. En primer lugar, que los conflictos son fenómenos relacionales, es decir, que no es posible comprender el conflicto si no abordamos ambos puntos de vista (Lederach, 1992); en nuestro estudio, las perspectivas de la empresa, de las comunidades y del Estado. En segundo lugar, el análisis del conflicto no se agota en la problemática, todo conflicto implica un problema, pero no todo problema genera conflicto. En un conflicto la problemática es una construcción intersubjetiva donde participan las partes; por tanto, es indispensable analizar la comprensión de ambos actores sobre el problema central y cómo estos influyen en sus percepciones (Mitchell, 2010). En tercer lugar, el desarrollo de las crisis se explica por las dinámicas de relacionamiento entre las partes, que son acompañadas de construcciones socioemocionales que se instauran en las partes a nivel individual, grupal y colectivo (Pruitt, Rubin y Kim, 2004).

\section{Resultados}

\section{Procesos comunicativos y recursos de movilización}

Se encontró que muchos de estos conflictos no tuvieron la visibilidad ni la espectacularidad mediática que tuvieron conflictos socioambientales, debido a su poca envergadura, al limitado repertorio de recursos contenciosos (Tilly y Tarrow, 2015) y al tipo de demandas, que estaban centradas mayormente en valores posmaterialistas (Inglehart, 2008), muy diferentes a demandas por contaminación de metales pesados o afectaciones a medios de subsistencia que suelen estar presentes en los conflictos socioambientales.

Las demandas en estos casos urbanos pasan por lo que Lederach (1992) denomina "desacuerdo responsable"; las acciones contenciosas tienen un cariz pacífico y están encausadas en los procedimientos legales. Por ejemplo, en el conflicto de Miraflores los vecinos escribieron 18 peticiones en oposición a la ordenanza de cambio de zonificación que se incluyeron en el expediente técnico. Sin embargo, las estrategias comunicativas no solamente son contenciosas, también sirven para generar adhesión y respaldo a las demandas y causas sociales; por ejemplo, en el caso de Miraflores el líder de la protesta, Fernando Taboada, repartió 600 cartas a los vecinos informando del problema y solicitando sus firmas para enviar la petición al alcalde (16 de febrero del 2015). De hecho, las estrategias comunicativas 
enmarcan sus acciones para configurar creencias y sentidos que inspiren y legitimen las actividades y campañas de los actores (Snow y Benford, 1992).

Las estrategias comunicativas utilizadas, como hemos visto, contribuyen sustantivamente en la ampliación de las dimensiones del conflicto. A decir de Mitchell (2010), el conflicto crece en el número de adherentes y pasa de la dimensión local a la dimensión metropolitana. Por otra parte, en los conflictos también surgen grupos secundantes que brindan apoyo a las partes en disputa, cumpliendo funciones de contraactivismo o de abogacía (Laue, 1982); los grupos que apoyan los proyectos en el caso de construcciones urbanas no se mostraron muy activos en lo comunicativo.

En el caso de Miraflores los que apoyan al proyecto de los jesuitas y de la constructora, basan su apoyo en su credibilidad en la institución religiosa porque son miembros de la parroquia. De hecho comprenden las necesidades de los jesuitas de obtener recursos para solventar la formación de los seminaristas.

En el caso del conflicto de San Isidro (caso 3), un sector de la población accedió a los medios masivos para plantear sus intereses y posiciones lo cual obligó a los representantes del municipio a hacer lo mismo; también pusieron banderolas en sus ventanas y balcones pero sin desarrollar acciones públicas organizadas. $\mathrm{Su}$ acceso a los medios les permitía que la visibilidad mediática del conflicto diera pie a una visibilidad pública (institucional) del conflicto, esperando con ello doblegar la decisión del gobierno local.

En el conflicto por la ampliación del tercer carril en Magdalena, los vecinos recurrieron a diversas estrategias, en primer lugar a las marchas, luego a la celebración del Día Mundial del Clima, a la organización de una marcha fúnebre por los árboles cortados, y luego, a acciones de vigilancia, haciendo turnos y pidiendo permisos en sus trabajos para resistir activamente a la acción municipal, pero evidenciando una mayor creatividad y un repertorio más amplio de recursos que generaban visibilidad y al mismo tiempo cohesión interna.

\section{Medios sociales como recursos de movilización en el conflicto}

Los medios sociales como Facebook y Twitter son frecuentemente usados para cumplir varias funciones en los procesos de conflictividad. En primer lugar, los usan las instituciones para comunicar información a los vecinos sobre los proyectos o, como en el caso de Miraflores, para aclarar las características técnicas.

El uso de medios sociales encaja muy bien en las características participativas propias de los vecinos, pues permitió que muchos de ellos expresasen a través de una fanpage de Facebook su oposición a los proyectos, motivando el activismo y la participación en el conflicto. 
En el caso de San Isidro, en lugar de crear una página propia, los grupos opositores al proyecto optaron por usar grupos de Facebook ya posicionados en contra de la gestión del alcalde vigente (Alerta San Isidro y San Isidro Libertad, ver anexo ${ }^{4}$ ) Esta fue la estrategia más viable para ellos pues aprovechaban que estas páginas ya tenían numerosos seguidores que además los articularon con otras iniciativas de grupos locales.

En el caso de Magdalena los grupos de residentes emplearon medios digitales intensamente para generar cohesión interna y para implementar sistemas de alerta temprana en contra de la intervención de las autoridades. Ellos se mantenían comunicados de través de mensajes de texto y de WhatsApp para coordinar acciones, turnarse en la vigilancia y hacer intervenciones rápidas en contra de los operadores y contratistas de la municipalidad.

Los vecinos de Miraflores y Magdalena se encontraban desvinculados de las organizaciones formales de participación que son las juntas vecinales. Pero a pesar de la falta de organicidad del activismo vecinal, el uso de medios sociales logra niveles de articulación y de respuesta al problema que sería muy difícil alcanzar sin su presencia, constituyéndose en recursos indispensables para la movilización en los conflictos de este tipo.

La difusión de sus puntos de vista (causas del conflicto, culpables y posibles soluciones) es otro de los usos sociales de Facebook y Twitter, ampliando así el número de adherentes (Snow y Benford, 1992) y visibilizando los acontecimientos desde un enfoque de victimización.

\section{Visibilidad del conflicto y recursos comunicativos}

Cuando los vecinos no obtienen respuesta de las autoridades o empresas, buscan visibilizar el conflicto primero en el espacio local, a través de pancartas colgadas en las ventanas de casas o edificios. Esto genera una ampliación del problema involucrando a vecinos que no están relacionados directamente con los líderes de las protestas.

Posteriormente, sea por iniciativa de los medios o por gestiones de los líderes, algunos medios cubren los conflictos dándoles una mayor visibilidad y consiguiendo la atención pública sobre los problemas sustantivos. Sin embargo, la cobertura de los conflictos urbanos de este tipo no llega a constituirse en una agenda nacional ni a permanecer en el tiempo, como sí ocurre con los conflictos socioambientales emblemáticos. De hecho, el conflicto de Miraflores solo fue cubierto por dos medios impresos: La República y Semana Económica.

4 El anexo puede consultarse en el siguiente enlace: https://drive.google.com/file/d/1jaerNz0Xnacu4uLtmOcH-LBbbDeTCD6/view 
En el caso del conflicto en Magdalena, la visibilidad mediática acompaña el surgimiento del conflicto, las obras se inician con el talado de los árboles de la avenida, lo cual genera la confrontación con los vecinos que atrajo a los medios y, debido al escándalo, los funcionarios decidieron suspender la tala. En este caso la presencia mediática funciona como un disuasivo para que el gobierno local suspenda las acciones contenciosas.

En los casos de San Isidro y Magdalena, en la medida en que, desde un inicio el conflicto se mediatizó, esto generó automáticamente una respuesta de las autoridades en los medios, en un caso para defender sus ideas y propuestas, como en San Isidro, y en el caso de Magdalena para deslegitimar a los opositores y sus reclamos, calificándolos de violentos, revoltosos y falsos ambientalistas, buscando anular a los mensajeros y a sus mensajes, generando con ello una dinámica de ida y vuelta de desprestigio y estereotipación.

\section{Comunicación institucional y conflictos por la residencialidad}

Por su parte, la empresa afrontó los conflictos y la crisis con el silencio; en el caso de Miraflores, la empresa arrendataria de los terrenos se negó a dar información a los medios sobre el proyecto, limitando las comunicaciones a los aspectos administrativos y técnicos, pues el consorcio empresarial dio una charla informativa el 21 de febrero del 2015, a cargo de la arquitecta que elaboró el proyecto, sobre el diseño técnico, sin permitir a los vecinos plantear sus objeciones, preocupaciones o temores. Por su parte, la Compañía de Jesús se limitó a publicar en su página de Facebook y a opinar en el sermón de la misa, llegando a una porción pequeña de los vecinos, los que se encuentran adscritos a ellos y a su entorno, sin establecer diálogos con la mayoría de vecinos.

Estas estrategias comunicativas evidencian que los actores institucionales no consideran los aspectos blandos de la comunicación como la construcción de confianza, y solo brindan información despersonalizada, sin desarrollar procesos comunicativos relacionales que alivien los temores o implementen mecanismos comunicativos que den garantías para que los problemas sean resueltos y se atiendan las demandas.

Al igual que en los conflictos socioambientales, las empresas se "escudan en la información técnica y en los datos fácticos a fin de cumplir con las formalidades legales o institucionales" (Ormachea, Caravedo, Moreno y Bedoya, 2014, p. 92), y emplean un esquema de comunicación corporativa que tiene como objetivo cuidar la reputación empresarial; por ello suprimen al máximo el contacto y la comunicación con la población. Este tipo de gestión comunicativa no funciona en situaciones de conflictividad, donde se requiere un mayor flujo de 
comunicación y la reconstrucción del tejido comunicativo dañado por las acciones coercitivas (Lederach, 1998).

El municipio de San Isidro, sin tratarse de una empresa, desarrolló similares procesos comunicativos en el conflicto por la remodelación de la calle Los Libertadores. Sustentó la legitimidad del proyecto de remodelación con base en la realización de una encuesta que no fue respondida por la mayoría de vecinos (solo el 27 \%), es decir, que se basó en procedimientos formales y poco interactivos para construir los consensos. A pesar de que el proyecto parece beneficiar al espacio público en diversos aspectos, su apego a los procesos formales y, por tanto, la ausencia de mecanismos y espacios de recojo de opiniones les dificultó la incorporación de cambios y mejoras al diseño del proyecto que incrementarían la aceptabilidad y reducirían la intensidad del conflicto.

Por tanto, estamos frente a una institucionalidad centrada en los procedimientos y apegadas a los trámites formales, que no recoge las preocupaciones de y afectaciones a los vecinos, sino que se concentró en las mejoras que sus iniciativas proponen a través de sus proyectos de inversión. El análisis de sus pronunciamientos y acciones muestra que no incorporan estrategias comunicativas para construir consensos, como señalan Saunders y Slim (2000), hay poca predisposición a escuchar o a incluir las inquietudes de los otros en su propia perspectiva, lo cual afecta en el mediano plazo a la gobernabilidad.

\section{Problemas subyacentes de los conflictos por la residencialidad}

Mitchell (2010) subraya que en la raíz de la recurrencia de los conflictos en un determinado periodo de tiempo, suelen existir condiciones estructurantes que generan su surgimiento constante. Uno de los problemas estructurantes reside en la normatividad que regula los cambios en el uso del territorio: la ordenanza metropolitana N. $^{\circ} 1617$ estipulaba la necesidad de una consulta vecinal para aprobar los cambios de zonificación. La nueva ordenanza n. ${ }^{\circ} 1911$ le da potestad al gobierno metropolitano para decidir sobre los cambios, reduciendo el poder de la voluntad de los vecinos y la opinión técnica de los gobiernos distritales.

Uno de los problemas en común en los casos estudiados ha sido la alteración de la residencialidad, entendida esta como la baja intensidad del uso del territorio destinado al hábitat, mientras que las inversiones tendían a intensificar el uso del territorio.

En el caso de Miraflores, el cambio de zonificación actual, de tipo "educativo" a una de tipo "comercio zonal" es el problema central, debido a que este cambio, según los vecinos, implicaría una mayor congestión vehicular en la zona, la presencia de ruidos molestos, una mayor inseguridad e intranquilidad permanente. 
Por lo tanto, estaría en juego la calidad de la residencialidad. La empresa, por su parte, sostiene que la presencia del hotel significaría el incremento del valor de los predios, pero en este caso la "promesa de beneficios económicos" no surte el mismo efecto que en las comunidades andinas, en tanto los residentes de la zona 10-C priorizan las condiciones residenciales de su hábitat por encima de los beneficios económicos o, en todo caso, no requieren mejorar su condición económica.

En el caso de Magdalena también se observa una afectación directa a la residencialidad, pues el incremento de carriles implicaría el flujo de 400 autos por hora en hora punta, con la consecuente contaminación sonora y de $\mathrm{CO}_{2}$, y la reducción de las áreas verdes a menos de $9 \mathrm{~m}^{2}$, por debajo de los estándares de la Organización Mundial de la Salud, todo lo cual afecta el valor del predio. Se trata de un caso de decisiones públicas que no favorecen directamente a los vecinos del espacio público en disputa, sino que se hace en nombre de un bien mayor que es reducir la congestión vehicular y facilitar el tránsito de los autos en hora punta, que en verdad beneficia a los automovilistas que usan el distrito para trasladarse a otros lugares en desmedro de la residencialidad de los vecinos.

El caso de San Isidro, por su parte, es un tema más complejo: el proyecto parte de cosmovisiones sobre el uso del espacio público que podríamos calificar de posmaterialistas, usando el concepto de Inglehart (2008), que prioriza el ornato, la humanización y las prácticas saludables en los espacios públicos, pues el proyecto fomenta el uso de las bicicletas, la recuperación de espacios verdes y una menor contaminación por $\mathrm{CO}_{2}$. En cambio, un sector de los vecinos estaba molesto porque la reducción de la vía pública y la construcción de la ciclovía en la calle Los Libertadores reducía los espacios para el estacionamiento de sus vehículos, para la salida de sus cocheras y para recibir a los diferentes servicios domiciliarios. En esencia, ellos no querían cambios, basados en su concepto tradicional de residencialidad que consiste en la apropiación del espacio público para un uso privado e individual. Buena parte de los que se oponían eran adultos mayores y no percibían los beneficios de espacios públicos más saludables y como lugares de encuentro y comunicación.

Parte del problema también consistió en que el municipio no compartió adecuadamente el diagnóstico de los problemas, conformándose con que el hecho de ganar las elecciones significaba la legitimación de sus planes de gobierno y sus premisas. Sin embargo, como es sabido, en los procesos electorales, especialmente los locales, los asuntos programáticos no tienen la atención debida. El diagnóstico que manejaba el municipio era que las calles se habían convertido en cocheras públicas de las diferentes oficinas que existen en el distrito, pero no era compartido por los vecinos (que entendían la residencialidad como control privado del espacio público). Solo después del surgimiento del conflicto, el municipio inició procesos informativos para compartir su diagnóstico y su visión de cambio. 


\section{Gestión comunicativa de los actores gubernamentales en los conflictos urbanos}

Generalmente en los conflictos socioambientales de envergadura, la presencia de actores terciarios que buscan salidas negociadas ocurre cuando los mecanismos y las reglas de juego no contemplan procesos participativos o estos son percibidos como injustos por una de las partes, o simplemente las acciones coercitivas desplegadas por las partes generan un entrampamiento o niveles de ingobernabilidad.

En los casos observados la presencia de terceros es casi nula, en parte porque existen mecanismos y procedimientos que encauzan el conflicto, en parte porque las estrategias contenciosas no llegan a niveles de violencia que amenacen la gobernanza territorial. Sin embargo, los actores que están llamados a desempeñar un rol imparcial no asumen el rol de mediadores, toman parte por los actores empresariales e incluso, como el municipio provincial de Lima, que cambió las reglas de juego a favor de las empresas y modificó el reglamento que exigía la consulta vecinal como requisito comunicativo y participativo dándole a la consulta un carácter "no vinculante", generan un incremento de las tensiones por parte de los vecinos.

En el caso de San Isidro, el órgano de control institucional del municipio en dos oportunidades, emitió información que cuestionaba los aspectos técnicos y procedimentales del proyecto, que sirvió de argumento a los opositores para cuestionar la remodelación, pero, al igual que en los otros casos, fue desoída por el alcalde y sus funcionarios.

Por su parte, la municipalidad de Miraflores retiró los carteles que los vecinos pusieron en el malecón en un esfuerzo por invisibilizar el problema. Aunque apoyaron la causa de los vecinos oponiéndose al cambio de zonificación, su accionar contra la expresión del conflicto no contribuyó a la distención.

El manejo de la comunicación del municipio de Magdalena fue más precario. Los vecinos se enteraron del proyecto debido al anuncio de la poda de árboles de la avenida por parte de la municipalidad y la tala consecuente el 28 de octubre del 2015, lo que generó una confrontación. Posteriormente surgieron denuncias de hostigamiento, multas a los vecinos opositores, generando un efecto cohesionador en ellos.

El municipio de Magdalena tuvo un rol activo en generar el escalamiento del conflicto al emplear tácticas pesadas para disuadir a los vecinos, quienes reportaron que se introdujeron "matones" en buses a las 3:00 am para garantizar el desarrollo de las obras, lo cual, ciertamente, generó un endurecimiento de las posiciones de los vecinos y derivó en un enfrentamiento. Además, el municipio anilló 
los árboles (corte de la corteza para acelerar su deterioro) lo cual fue interpretado por los vecinos como una agresión más a su resistencia a la tala de árboles.

En el caso del conflicto por la ampliación del tercer carril en Magdalena, la confrontación entre el proyecto de la municipalidad y los vecinos no permitió una instancia de apelación o la participación de un tercero imparcial pues la autoridad estaba parcializada (parte primaria); por ello los vecinos recurrieron a la Defensoría del Pueblo, al Congreso de la República y al Poder Judicial, aunque esta vía es engorrosa y lenta pero la única capaz de revertir la decisión del Gobierno local.

La estrategia de persuasión a través de un uso intensivo de medios informativos fue más intensa en el caso del municipio de San Isidro. Instalaron casetas con paneles informativos, desarrollaron videos que colgaron en sus propias redes sociales, sección de "preguntas frecuentes" en su sitio en internet, comunicados y visitas casa por casa para explicar los beneficios de la remodelación. Aunque ninguno de estos medios fue interactivo, salvo las visitas domiciliarias, abordaron la problemática de manera individual sin atender las demandas colectivas, pues, además, el alcalde no atendió a los vecinos que asistieron una reunión de discusión sobre los problemas vecinales.

El análisis de los roles desempeñados por los gobiernos locales evidenció la carencia en estas instituciones de estrategias comunicativas para distender el conflicto, o de procesos comunicativos que construyan confianza. Más bien observamos que el uso de estrategias comunicativas ambiguas y sin transparencia abonó la polarización y el incremento de la oposición a los proyectos de inversión urbanos y en uno de los casos estudiados el uso de acciones coercitivas quebró la comunicación e instauró una barrera emocional y cognitiva para el diálogo (Pruitt, Rubin y Kim, 2004). En resumen, la gestión comunicativa de los conflictos tendría una influencia en el curso desencauzado que los conflictos toman, y por tanto, no estarían predeterminados por el problema estructural, lo cual explicaría las variaciones observadas en los casos. Esta afirmación requiere del análisis de un mayor número de casos para poder comprobarse con mayor precisión.

\section{Contribución}

El crecimiento urbano y el boom de la construcción impulsado por el neoliberalismo en un corto periodo de tiempo, tiene un efecto de "modernización acelerada" que moldea, aunque no determina el surgimiento de los conflictos urbanos por los cambios en las condiciones de residencialidad. Diversos actores vecinales generan resistencias a la reconfiguración de los servicios urbanos debido a que ven amenazados su acceso, control o distribución de los recursos urbanos que satisfacen sus necesidades de residencialidad. 
Evidenciamos que la residencialidad es un elemento que no se discute entre autoridades, empresas y población. Existen diferentes cosmovisiones en torno a la residencialidad. De un lado, cosmovisiones que incluyen valores posmaterialistas basados en la salud, la humanización del espacio público y el cuidado del medio ambiente, como en los casos caso de la población de Magdalena y de Miraflores y del municipio de San Isidro. Del otro lado tenemos cosmovisiones tradicionales basadas en el uso del espacio público como el acceso individual a servicios urbanos o el tránsito vehicular en los casos de la empresa y el municipio provincial de Lima en el conflicto de Miraflores, los residentes de San Isidro y el Municipio de Magdalena.

Estas diferentes visiones de desarrollo no son consensuadas por las autoridades o empresas, y permiten que afloren subyacentemente con los conflictos. Precisamente requieren de espacios de diálogo y comprensión mutua, pero conforme el conflicto se instaura esta posibilidad se dificulta. Los actores generan su práctica en base a la información de la que disponen y en la medida en que no se constituyen espacios de interacción y diálogo, la información no fluye entre las partes confrontadas; por tanto, no es posible la convergencia de intereses y la comprensión de las necesidades de los actores. Por ello prevalecen las posiciones y los actores se enfocan en desprestigiar al otro y a sus ideas, lo cual incrementa la polarización y debilita el diálogo.

Por lo analizado, el esfuerzo que realizaron las instituciones para desarrollar procesos comunicativos ex ante fue exiguo; no encontramos acciones que busquen compartir la información mutua para crear consensos en torno al diagnóstico de los problemas y sus posibles soluciones.

Las campañas comunicativas se inician - si es que existen- cuando los conflictos ya se instauraron entre las partes y la consiguiente desconfianza y brecha emocional impide - aunque no imposibilita del todo- una comunicación genuina que, según Caravedo y Moreno (2007) consiste en una disposición a escuchar y comprender las necesidades de las otras partes.

Encontramos, por tanto, que las condiciones estructurales de los conflictos urbanos en torno a la modificación de la residencialidad se encuentran mediadas por la gestión comunicativa empresarial o gubernamental o el enfoque coercitivo con que se desarrollan las estrategias comunicativas que, en lugar de canalizar el conflicto, estimulan una mayor confrontación. Esto significa que la relación entre causas y conflicto no es directa sino que depende de factores como la cosmovisión y la gestión comunicativa, entre otros.

Sin embargo, a diferencia de los conflictos laborales, por ejemplo, la normatividad que regula la comunicación y la participación con los vecinos es muy precaria y no garantiza la información debida; la calidad de los espacios de 
discusión contribuye a una gestión opaca del territorio e instaura la desconfianza y agudiza los conflictos.

El análisis que hacen Calle y Ryan (2016) de 24 casos de conflictos socioambientales en Latinoamérica encuentra que en solo siete de ellos los procedimientos fueron eficaces para integrar las observaciones de la población. En los casos observados la movilización de los vecinos de zonas residenciales influyó en la paralización total o parcial de las obras, doblegando la voluntad de la autoridad. La visibilidad mediática de los conflictos desarrollada por los vecinos fue clave para este cambio en la voluntad política.

De otro lado, en los casos observados vemos cómo los vecinos se oponen a los cambios que los afecten en sus condiciones de residencialidad; estamos, por tanto, frente a casos "NIMBY" (Not in my back yard, "no en mi patio"), donde si bien apoyan el desarrollo urbano, la modernización y las reglas de juego neoliberales de la industria de la construcción y la modernización del espacio público, se oponen a estas cuando son afectados directamente.

Coincidimos con Calle y Ryan (2016) en que en los casos analizados, en la movilización concurren acciones colectivas de incidencia y una estructura participativa mínima (informativa, decisional, espacios, procedimientos), pero creemos que, dadas las características de estas poblaciones, con escasa cultura participativa y vecinal, el uso de medios digitales contribuye a una construcción socioemocional como sujetos colectivos; les sirve para desarrollar cohesión e identidad (Della Porta y Diani, 2010), para ampliar su campo de incidencia (Tarrow 2012; Tilly y Tarrow, 2015), y para incrementar su respaldo (Snow y Benford, 1992). Por tanto, los medios sociales están siendo sustantivos en la conflictividad urbana por la residencialidad. Sin embargo, no abonan al diálogo ni a la creación de espacios de interacción entre ambas partes del conflicto, sino que incrementan la conflictividad.

Concluimos que los repertorios comunicativos empleados por las partes en disputa (residentes o autoridades) están centrados en fortalecer su posición y poder en el conflicto, buscando impactar en otros actores para tener un mayor respaldo público a sus causas.

Las estrategias comunicativas no se diseñan para construir puentes, generar acercamientos o para el diálogo. Especialmente desde la acción gubernamental hace falta reenfocar la dimensión comunicativa en la gestión de los conflictos urbanos. 


\section{Referencias}

Alfaro, R. M. (2015). Una comunicación para otro desarrollo. Lima: A.C.S. Calandria.

Ariñez, N. (2007). Medios de comunicación social y conflictos. Aproximaciones al caso de Carlos Mesa. Revista Punto Cero, 13(15), 7-14. Recuperado de http:// www.redalyc.org/pdf/4218/421839607001.pdf

Avruch, K. (2013). Culture, Identity, Power and Practice. Boulder: Paradigm.

Bebbington, A. y Humphreys, D. (2009). Actores y ambientalismos: conflictos socio-ambientales en Perú. Iconos, 35, pp. 117-128. https://doi.org/10.17141/ iconos.35.2009.371.

Beltrán, L. R. (2006). Comunicación para el desarrollo en Latinoamérica: Un recuento de medio siglo. Anagramas Rumbos y Sentidos de la Comunicación, 4(8), 53-76. Recuperado de https://dialnet.unirioja.es/descarga/articulo/5181393.pdf

Burton, John. (2000). La resolución de conflictos como sistema político. Fairfax: Instituto de Análisis y Resolución de Conflictos (ICAR), George Mason University.

Calle, I. y Ryan, D. (2016). La participación ciudadana en los procesos de evaluación de impacto ambiental: análisis de casos en 6 países de Latinoamérica. Lima: Sociedad Peruana de Derecho Ambiental (SPDA).

Cáneva, V. (2015). Organizaciones autoconvocadas: construcción de lazos sociales urbanos en tiempos de restitución de lo público. Contratexto 23, pp. 93-110. Recuperado de https://revistas.ulima.edu.pe/index.php/contratexto/article/ view/413/395

Caravedo J. y Moreno G. (2007). Construcción y fortalecimiento de espacios de diálogo. Lima: ProDiálogo.

Castaño, A. (2009). Inserción social y residencialidad de los inmigrantes en las áreas urbanas de Sevilla y El Ejido. AREAS. Revista Internacional de Ciencias Sociales, 28, pp. 89-109. Recuperado de http://revistas.um.es/areas/article/ view/118761/112051

Castells, M. (1974). La cuestión urbana. Madrid: Siglo XXI Editores.

De Echave, J., Diez, A., Huber, L., Revez, B., Ricard, X., y Tanaka, M. (2009). Minería y conflicto social. Lima: IEP/CPICA/CBC/CIES.

Defensoría del Pueblo (2017). Ante todo, el diálogo: Defensoría del pueblo y conflictos sociales y políticos. Lima: Adjuntía para la Prevención de Conflictos Sociales y la Gobernabilidad.

Della Porta, D. y Diani, M. (2010). Social Movements: An Introduction. Malden: Blackwell Publishing.

De los Ríos, S. (2017). La recuperación de la residencialidad en la ciudad global. Caso: Centro Histórico de Lima, Perú. Medio Ambiente y Urbanización, 86(1), 
pp. 129-144. Recuperado de https://www.ingentaconnect.com/contentone/ iieal/meda/2017/00000086/00000001/art00006\#

Department for International Development (2000). Working with the Media in Conflicts and other Emergencies. Department for International Development. Londres.

Deutch, M. y Coleman P. (2012). Psychological Components of Sustainable Peace. Nueva York: Springer.

Díaz, B., Rojas, L. R., y Romero, S. (2005). Resolución pacífica de conflictos y gestión de cultura de paz en los medios de comunicación social venezolanos. Revista ORBIS 1(1), 52-63. Recuperado de https:/dialnet.unirioja.es/ descarga/articulo/2251152.pdf

Esser, F. y Strömbäck, J. (2014). Mediatization of Politics: Understanding the Transformation of Western Democracies. Londres: Palgrave Macmillan.

Eto, G. (2010). Bagua, un conflicto en Primera Plana (Tesis de magíster). Lima: PUCP.

Fisher, R. J. (2009). Interactive conflict resolution: Dialogue, conflict analysis, and problemsolving. En: Sandole, D. J. D., Byrne, S., Sandole-Staroste, I., y Senehi, J. (Eds.), Handbook of Conflict Analysis and Resolution, pp. 328-339. Abingdon: Taylor \& Francis e-Library.

García-Vargas, A.; Gaona, M. y López, A. (2016). Intersecciones: espacio físico, social y mediático en la construcción cotidiana de una "ciudad ordinaria" en San Salvador de Jujuy, Argentina. Comunicación y medios, 33, pp. 89-114. doi:10.5354/0719-1529.2016.37236

Gumucio, A. (2001). Haciendo olas: Historias de la comunicación participativa para el cambio social. Nueva York: The Rockefeller Fundation.

Hieber, L. (2001). Lifeline Media: Reaching population in crisis. A guide to developing media projects in conflicts situations. Suiza: Media Action International.

Huamán, L. (2012). Medios de comunicación en conflictos socio-ambientales: tratamiento periodístico del conflicto socio-ambiental Quellaveco (Tesis para optar el título de licenciada en comunicaciones). Lima: PUCP.

Huamaní, G., Macassi, S., Alegría, J., y Rojas, T. (2012). Hacia una mejor gestión de los conflictos socioambientales en el Perú. Lima: Consorcio de Investigación Económica y Social (CIES).

Inglehart, R. (2008). Changing values among western publics from 1970 to 2006. West European Politics, 31(1-2), pp. 130-146. https://doi. org/10.1080/01402380701834747

Kriesberg, L. y Dayton, B. (2012). Constructive conflicts: From escalation to resolution. Maryland: Rowman \& Littlefield Publishers, Inc. 
Laue, J. H. (1982), Ethical Considerations in Choosing Intervention Roles. Peace $\mathcal{E}$ Change, 8, pp. 29-41. https://doi.org/10.1111/j.1468-0130.1982.tb00646.x

Lederach, J. P. (1992). Enredos, pleitos y problemas: Una guía práctica para ayudar a resolver conflictos. Ciudad de Guatemala: Semilla.

Lederach, J. P. (1998). Construyendo la paz. Reconciliación sostenible en sociedades divididas. Bilbao: Gernika Gogoratuz.

Macassi, S. (2011). Los medios de comunicación en el ciclo de vida de los conflictos: Aportes de la comunicación a la gobernabilidad en contextos de crisis. Contratexto 19, pp. 35-52. Recuperado de http://revistas.ulima.edu.pe/index. php/contratexto/article/viewFile/183/159

Martínez Alier, J. (2009). El ecologismo de los pobres: conflictos ambientales y lenguajes de valoración. Barcelona: Icaria.

Mitchell, C. (2010). The Structure of International Conflict. London: Palgrave McMillan.

Mitchell, C. (2016). La naturaleza de los conflictos intratables. Resolución de conflictos en el s. XXI. Barcelona: Institut Català Internacional per la Pau.

Monterrubio, A. (2011). Políticas habitacionales y residencialidad en el Centro Histórico de la Ciudad de México. Argumentos, 24(66), pp. 37-59. Recuperado de http://www.scielo.org.mx/scielo.php?script=sci_arttext\&pid=S018757952011000200003\&lng=es\&nrm=iso

Obar, J. y Wildman, S. (2015). Social media definition and the governance challenge: An introduction to the special issue. Telecommunications Policy, 39(9), pp. 745-750. http://dx.doi.org/10.2139/ssrn.2647377

Ormachea, I.; Caravedo, J.; Moreno, G. y Bedoya, C. (2014). Minería, conflicto social y diálogo. Lima: ProDiálogo.

Pruitt, D., Rubin, J., y Kim, S. (2004). Social conflict. Escalation, stalemate, and settlement. Nueva York: McGraw-Hill.

Reguillo, R. (2005). Ciudad riesgo y malestares. Hacia una antropología del acontecimiento. En: García-Canclini, N. (Coord.), La antropología urbana en México, pp. 307-340. México: Fondo de Cultura Económica.

Rubenstein, R.; Botes, J.; Dukes, F. y Stephens, J. B. (1994). Frameworks for interpreting conflicts. A handbook for journalists. Fairfax: Instituto de Análisis y Resolución de Conflictos (ICAR)/George Mason University.

Sandoval, V. (2015). Apuntes sobre periodismo y conflictos socioambientales en Bolivia: El caso del TIPNIS. En: Macassi, S. y Acevedo, J. Confrontación y diálogo: Medios y conflictos en los países andinos. Lima: Pontificia Universidad Católica del Perú, Fondo Editorial. 
Saunders, H. y Slim. R. (2000). Diálogo para cambiar las relaciones conflictivas. Fairfax: Instituto de Análisis y Resolución de Conflictos, George Mason University.

Silva, A. (2007). Imaginarios urbanos en América Latina: Urbanismos ciudadanos. Barcelona: Fundació Antoni Tàpies.

Simancas, M. y García, J. (2012). La residencialidad: ¿la alternativa a la oferta alojativa extrahotelera de Canarias? Turismo, 3-4. 13-21. Recuperado de https:/dialnet. unirioja.es/servlet/articulo?codigo $=5641772$ \&orden $=0$ \&info $=$ link

Snow D. y Benford, R. (1992). Master frames and cycles of protest. En: Morris, A., y Mueller, C. (Eds.), Frontiers in Social Movement Theory, pp. 133-155. New Haven: Yale University Press.

Tarrow, S. (2012). Strangers at the Gates: Movements and States in Contentious Politic. Cambridge University Press.

Tilly, C. (2006). Regimes and Repertoires. Chicago: University of Chicago Press.

Tilly, C. y Tarrow, S. (2015). Contentious Politics. Oxford: Oxford University Press.

Torres, Y. (2007). 11 de enero en Cochabamba: De los medios, miedos y otros demonios. Lazos 2(4), pp. 18-22. Recuperado de https://issuu.com/ unirbolivia/docs/revista-lazos-04

Torrico, E. (2009). Los medios de comunicación masiva en conflicto: Reflexiones sobre el papel del periodismo en la tensa situación boliviana. Contratexto, 17, pp. 13-20. Recuperado de http://fresno.ulima.edu.pe/sf/sf_bdfde.nsf/ OtrosWeb/CONT17TORRICO/\$file/01-contratexto17\%20TORRICO.pdf

Tufte, T. (2015). Comunicación para el cambio social. La participación y el empoderamiento como base para el desarrollo mundial. Barcelona: Icaria.

Vallacher, R. R., Coleman, P., Nowak, A., Bui-Wrzosinska, L., Liebovitch, L. S., Kugler, K., y Bartoli, A. (2013). Attracted to Conflict. Dynamic Foundation of Destructive Social Relations. Londres: Springer.

Villegas, S. (2007). Conflictos sociales en la escena televisiva (ponencia presentada al IV seminario latinoamericano de investigación de la Comunicación). La Paz, 8 al 10 de noviembre del 2007. Recuperado de http://www.redalyc.org/ articulo.oa?id=199520934008

Yáñez, L. (2014). Crecimiento urbano continuará con edificios con el doble o el triple de altura. Punto Edu. PUCP. Recuperado de https://puntoedu.pucp. edu.pe/noticias/boom-inmobiliario-y-urbanismo/ 